\title{
Feasibility, Acceptability, and Clinical Trends of a Mindfulness-Informed Child Welfare Intervention: Implications for Trauma-Informed Practice
}

\author{
Samantha M. Brown \\ Jennifer Bellamy
}

\begin{abstract}
Exposure to stress and early life trauma have been linked to child maltreatment and parental substance misuse. These issues often co-occur, yet few child welfare services target their shared underlying causes in a single intervention. Teaching mindfulnessinformed strategies to substance-misusing families in the child welfare system may be one promising trauma-informed approach. As part of a larger pilot study testing the initial efficacy of a mindfulness-informed intervention for parents in public child welfare, this study explored the feasibility, acceptability, and clinical trends of the intervention using weekly reports of stress, coping, and mindfulness. Findings show support for the feasibility and acceptability of the intervention as well as positive responses to the intervention on measures of stress and mindfulness. However, the impact of the intervention varied with regard to improving weekly coping among participants. Implications for the integration of mindfulness into child welfare practice as a trauma-informed approach are discussed.
\end{abstract}

Keywords: Child welfare, coping, mindfulness, stress, trauma

Early adversity can impact multiple domains of an individual's life. Two pernicious, and frequently co-occurring risk factors, often linked to childhood adversity are maltreatment and parental substance misuse. Approximately one-half to two-thirds of child welfare cases involve parental substance misuse (National Center on Addiction and Substance Abuse at Columbia University, 2005). The consequences of concomitant parental substance misuse and child maltreatment are significant. Parents with substance misuse tend to remain involved in the child welfare system longer (U.S. Department of Health and Human Services, National Clearinghouse on Child Abuse and Neglect, 2003) and have an increased likelihood of termination of parental rights (Harris-McKoy, Meyer, McWey, \& Henderson, 2014). However, child welfare services tend to focus on addressing the symptoms of substance misuse and parenting, rather than their shared underlying causes, including parental stress and trauma. Child welfare-involved parents have experienced their own histories of trauma as a population (National Child Traumatic Stress Network, 2011). For example, Oliver (1993) found that approximately one-third of parents who maltreated their children have experienced abuse in childhood.

Although trauma-informed services have become more common in child welfare, substance misuse treatment programs and parenting services tend to be delivered to parents in silos. Parents' own trauma histories, in combination with additional stressors associated with the demands parents must meet in the context of child welfare, highlight the need to develop practice approaches that are trauma-informed and that target the underlying mechanisms implicated in both substance misuse and maladaptive parenting. Mindfulnessinformed programs may be one promising approach that offers this support to families

Samantha M. Brown, PhD, MA, LPC is a Postdoctoral Fellow (T32 MH015442), School of Medicine, University of Colorado, Aurora, CO 80045. Jennifer Bellamy, PhD is an Associate Professor, Graduate School of Social Work, University of Denver, Denver, CO 80208. 
using a trauma-informed perspective (Follette, Palm, \& Pearson, 2006). Mindfulness has been used with a variety of groups with high levels of trauma, including child abuse survivors (Kimbrough, Magyari, Langenberg, Chesney, \& Berman, 2010) and combat veterans (King et al., 2013).

Mindfulness is defined as a nonjudgmental acceptance and awareness of present moment experiences (Bishop et al., 2004; Kabat-Zinn, 1994). It has been conceptualized by an awareness of present moment experience with a quality of curiosity, and by the ability to decenter or distance oneself from unpleasant thoughts and feelings without being carried away by them (Lau et al., 2006). Teaching mindfulness to families aligns with traumainformed child welfare practice such that strategies can be used to help target stress and coping with the potential to improve long-term parent-child interactions and family functioning. For example, individuals exposed to adversity may struggle to adaptively cope, thereby affecting self-regulation skills (Cloitre et al., 2009) and engaging in more avoidant-related coping (Benotsch et al., 2000) in the presence of distressing thoughts and feelings. Exposure to stress and impaired coping may in turn increase the likelihood of unhealthy behaviors such as substance use or hostile parenting practices.

Cultivating mindfulness may help to counteract the effects of previously avoided internal experiences as well as habitual, impulsive behaviors (Kabat-Zinn, 1990). In a review, mindfulness has been shown to buffer against maladaptive behaviors and, instead, replace these behaviors with controlled coping responses (Weinstein, Brown, \& Ryan, 2009). In addition, because experiencing adversity may result in avoidant-related behaviors subsequently impacting parent participation in treatment (Littell, Alexander, \& Reynolds, 2001; Littell \& Tajima, 2000), promoting acceptance and awareness through mindfulness may also positively affect parents' engagement in child welfare services more broadly. Prior research demonstrates that mindfulness-based interventions are effective in reducing stress (Carlson, Speca, Patel, \& Goodey, 2004; Williams, Kolar, Reger, \& Pearson, 2001), trauma symptoms (Kimbrough et al., 2010; King et al., 2013), and substance misuse (Bowen et al., 2009), as well as improving parent-child interactions (Duncan, Coatsworth, \& Greenberg, 2009a).

Despite progressive efforts to improve outcomes for child welfare-involved families with substance misuse, few programs target stress-precipitated child maltreatment and concomitant substance misuse in a single intervention. As such, this study is part of a larger pilot study testing the initial efficacy of a mindfulness-informed intervention for parents in public child welfare. The goals of this study were to pilot a new mindfulness-informed child welfare intervention and explore the feasibility and acceptability as well as assess participants' response to the intervention using weekly clinical reports of stress, coping, and mindfulness. 


\section{Method}

\section{Sample and Procedure}

This study was conducted in an urban area in the rocky mountain region of the United States. The sample of participants were recruited by child protection caseworkers and health department nurses. Caseworkers and nurses referred parents for participation in the mindfulness program if the following criteria were met: 1) the family was involved in, or at risk for involvement in, child welfare, 2) substance misuse was a presenting concern, and 3) children remained in the home with parents or parents had weekly visitation with their children. The caseworkers and nurses gave recruitment flyers to eligible parents, and the parents were instructed to release their names and phone numbers to the principal investigator if they were interested in participating in the research study. Participants were then contacted by phone by the principal investigator to confirm eligibility and consent to participation. The study procedures were approved by the authors' Institutional Review Board.

The mindfulness program implemented in the present study was delivered to parents in six weekly, in-home sessions, lasting approximately one-hour. The first 5-15 minutes of the session included a weekly check-in about participants' stress experiences and use of mindfulness-informed strategies. Next, participants were provided with psychoeducational content on issues related to stress, triggers, parenting, and the use of mindfulness techniques to address these domains. The last 15 minutes of the session included an experiential mindfulness-informed exercise, which participants could use in their day-today lives. Participants also completed brief questionnaires in each session that assessed stress, coping, mindfulness, and their reaction to the session content. In addition, at the conclusion of the six-session intervention, participants completed a program satisfaction questionnaire to assess their experiences and thoughts regarding the acceptability of the program.

Sessions were developed and delivered by a masters-level, licensed counselor. In contrast to traditional cognitive-behavioral therapies, it is recommended that providers delivering mindfulness are trained in implementation and engage in their own mindfulness practice (Segal, Williams, \& Teasdale, 2002). As such, this provider received specific training in mindfulness, which included approximately 24 hours of in-person training, consisting of didactic and experiential education and clinical skills practice, in addition to an online training on mindfulness developed by Kabat-Zinn (2015).

\section{Mindfulness-Informed Intervention for Child Welfare-Involved Parents}

The intervention included sessions on mindfulness (i.e., awareness of present-moment experiences), cognitive reappraisal (i.e., changing the meaning of emotionally triggering stimuli), and savoring (i.e., selectively focus on positive stimuli) that were adapted from an extant mindfulness program (Mindfulness-Oriented Recovery Enhancement; Garland, 2013) that has demonstrated positive effects on stress and substance use among adult populations (Garland, Gaylord, Boettiger, \& Howard, 2010), but has not been used with 
parents in child welfare. Additional sessions on stress, mindful parenting, and future planning were developed by integrating theory and research on parent-child relationships, family functioning, and mindfulness-based parenting (e.g., Duncan, Coatsworth, \& Greenberg, 2009b; Hillson \& Kuiper, 1994; Kabat-Zinn \& Kabat-Zinn, 1997; Patterson, 1982). In addition, in order to provide an individualized and flexible intervention to meet multiple familial needs and align with the constraints of the child welfare system, sessions were delivered in family's homes and session content was tailored to address each family's unique stressors and strengths. Sessions were delivered for approximately one hour as prior research indicates that standard 2.5-hour mindfulness-based sessions may be too long for vulnerable populations (Dutton, Bermudez, Matas, Majid, \& Myers, 2013). As such, the newly developed mindfulness program for child welfare-involved parents aimed to increase parents' awareness regarding the triggers associated with stressful situations and substance use, and provide insight into how automatic maladaptive patterns of coping may lead to dysfunctional parent-child interactions. Parents were taught both formal and informal strategies including mindful breathing, reappraisal, savoring of pleasant experiences, and mindful parenting such that they could pay attention to and self-regulate their own thoughts and feelings in present moment experiences, while also responding to their children in a conscious effort by attending to their child's needs. The content of each of the sessions are presented in Table 1.

\section{Table 1. Outline of Mindfulness-Informed Program}

\begin{tabular}{cl}
\hline Session \# & \multicolumn{1}{c}{ Training Sessions } \\
\hline 1 & Introduction to Mindfulness, Automatic Habits, and Maladaptive Behaviors \\
3 & Mindful Reappraisal \\
4 & Savoring Positive Experiences and Interactions with Children \\
5 & Understanding Impulses and Relationship to Stress \\
6 & Mindful Parenting \\
\hline
\end{tabular}

\section{Measures}

Demographics. A basic demographic questionnaire was administered to parents to capture parent characteristics as well as their mental health, substance use, and child welfare histories.

Stress. The Short Stress State Questionnaire (SSSQ; Helton, 2004) was used to measure weekly state stress. The SSSQ is a 24-item instrument comprised of three domains of stress (Distress, Worry, and Engagement). Only the Distress subscale was used in the present study. Since participants were taught skills that aimed to increase positive emotions under conditions of stress, the Distress subscale was used to measure changes in reports of negative affect-emotion. Participants were asked to rate statements on the degree to which they agree with how they felt during the past week $(1=$ not at all; $5=$ extremely $)$. Eight items comprise the Distress subscale, ranging in scores from 8-40. The SSSQ Distress subscale demonstrates high reliability (Cronbach's $\alpha=.87$; Helton, 2004).

Coping. Coping was examined using the Brief COPE (Carver, 1997). The Brief COPE includes 14 scales of two items each, which participants rate on a 4-point scale from 1 ( $I$ 
haven't been doing this at all) to 4 (I've been doing this a lot). To align with the reappraisal skills taught to participants in the mindfulness intervention, only the Positive Reframing subscale was used in this study. Therefore, scores range from 2-8. This subscale was hypothesized to assess changes in weekly coping, though prior reliability estimates of this subscale yield a questionable Cronbach's $\alpha$ of .60 (Yusoff, Low, \& Yip, 2010), perhaps due to the subscale being comprised of only two-item indicators.

Mindfulness. To assess state mindfulness that can vary in a short period of time, the Toronto Mindfulness Scale (TMS; Davis, Lau, \& Cairns, 2009) was used. Thirteen items comprise the two factors (Curiosity and Decentering) of the TMS. Mindful Curiosity refers to the awareness of present moment experiences with a quality of curiosity. Mindful Decentering captures how well participants are able to distance themselves from situations that may provoke unwanted thoughts or feelings. After engaging in a brief 5-10 minute mindful breathing and/or experiential exercise, participants were asked to rate items on a scale from 0 (not at all) to 4 (very much). Internal consistency reliability include a Cronbach's $\alpha$ of .88 for Curiosity and .84 for Decentering (Davis et al., 2009). Scores on the Mindful Curiosity scale range from 0-24 and on the Mindful Decentering scale from 028.

Feasibility. Intervention feasibility was determined by recruitment, retention, and treatment attendance rates among parents involved in the child welfare system with cooccurring substance use. Three measures of recruitment and treatment attendance were used for this study: 1) the number of participants enrolled in the intervention, 2) the number of participants who dropped out prior to completion of the post-intervention questionnaires, and 3) the number of intervention sessions attended.

Acceptability to Parents. To assess the acceptability of the mindfulness-informed intervention, we assessed participant's perspectives of the intervention, overall, as well as during individual sessions. With regard to the acceptability of each session, participants were asked four questions to assess their reaction to session content. Specifically, participants were asked to rate on a 5-point Likert-type scale $(1=$ strongly disagree; $5=$ strongly agree) if they gained something positive from participating in the session, if the session raised emotional issues they had not expected, if they gained insight about their experiences during the session, and if the session made them think about things they did not want to think about. These questions were selected in order to understand the degree to which each session helped participants cultivate awareness regarding their experiences within the session as well as identify whether session content may have been more detrimental to participant outcomes. These ratings were subsequently used to inform future adaptations to the intervention.

A program satisfaction questionnaire was also administered to assess the acceptability of the overall program. Participants completed 10 items (e.g., "The program I received was a big help to me"; "I got the kind of help through the program that I needed"; "I enjoyed learning about the concept of mindfulness"; "I would recommend this program to other families") in which they rated the way they felt about the services they received on a 5point scale $(1=$ none of the time; $5=$ all of the time $)$. Percentages of each of the 10 items were computed for analysis. The survey also consisted of five open-ended questions 
describing the benefits and challenges of participating in the intervention as well as recommendations for future iterations of the mindfulness sessions.

\section{Data Analysis}

Descriptive statistics (means, standard deviations, or percentages) were used to describe the sample characteristics, as well as the feasibility and acceptability of providing this intervention in the context of child welfare. This can be examined by describing the proportion of families retained and number of sessions attended, and by participants' reactions to session content and overall program satisfaction levels. To analyze the openended questions on the participant satisfaction questionnaire, a template approach (Crabtree \& Miller, 1999) was applied such that a priori codes were used that were associated with the strengths and weaknesses of the program. Specifically, these codes were used to address the open-ended program satisfaction questions that included the following: 1) What were the benefits of participating in this program?, 2) What were the drawbacks of participating in this program?, 3) What did you notice change in yourself since participating in this program?, 4) How could sessions be improved? and 5) What else would you like to add that relates to your experience while participating in this program? An iterative process was used to identify codes within these a priori categories and were subsequently grouped into themes. Two coders reviewed participant responses and percent agreement was calculated with high rates $(90 \%)$ of inter-rater reliability.

Participants' scores from the stress, coping, and mindfulness questionnaires were aggregated and their mean scores were calculated to evaluate weekly group-level trends of the mindfulness-informed intervention. Although primarily used for single-subjects research, a similar procedure to the Percentage of Nonoverlapping Data (PND) metric (Scruggs, Mastropieri, \& Casto, 1987) was used to understand weekly clinical trends. The PND metric is a systematic approach used in single-subjects research that produces a common outcome metric across participants, allowing for ease of data interpretation and providing meaningful information on whether or not a program is beneficial to participants.

To calculate the PND for this study, the proportion of data points in the treatment condition that exceeded or fell below the baseline value was identified. The intervention was designed to increase mindfulness and coping among participants, and therefore, the proportion of treatment data points that exceeded the baseline value was calculated to determine if the intervention had positive effects on these domains of functioning. In contrast, the intervention was designed to decrease participant stress. As such, the proportion of treatment values that fell below the baseline data point was calculated to identify reductions in stress.

According to guidelines established by Scruggs and Mastropieri (1998) for singlesubjects research, and which were applied to the aggregated mean scores in this study, PND scores greater than 90 indicate extremely successful treatments, scores that range from 70 to 90 represent successful treatments, scores from 50 to 70 are questionable, and scores less than 50 are often identified as unsuccessful treatments. For example, if half of the treatment data points in the current study for participant mean scores (3/6 sessions) do not exceed the baseline level mean score, then the PND score is 50\%, thereby indicating that 
the intervention was not beneficial to participants in a particular domain. In addition to calculating the PND statistics, participants' aggregated graphical data were displayed to provide visual insight into how intervention participants changed over time on outcomes of stress, coping, and mindfulness across each of the six weekly sessions.

\section{Results}

\section{Feasibility}

Over the recruitment period, 15 participants were selected to participate in the mindfulness-informed intervention. One parent selected to participate in the intervention dropped out prior to the start of the intervention and three others dropped out early in the program (after the second or third sessions) due to moving to another state, personal life changes and feeling overwhelmed, or being unreachable at subsequent contacts by the researcher. Thus, 11 of the 15 parents (73\%) were retained in the program. With respect to treatment attendance, on average, parents completed $5.8(S D=.40)$ sessions. The provider sent weekly reminders to participants and flexibly scheduled sessions, which may have positively contributed to study participation. The primary barrier to attending all six mindfulness sessions included frequent rescheduling due to other demands parents had to meet, such as attending other child welfare-mandated services or visitation with children.

\section{Sample Characteristics}

Eleven parents completed the mindfulness-informed intervention. Eight were mothers (72.7\%) and three were fathers $(27.3 \%)$ who identified racially and/or ethnically as White $(n=9 ; 81.8 \%)$, Black $(n=1 ; 9.1 \%)$, and Puerto Rican $(n=1 ; 9.1 \%)$. Participants averaged $33.18(S D=11.05)$ years in age, ranging from 21 - to 53-years-old. Slightly less than half of the sample were employed $(n=5 ; 45.5 \%)$ and resided within lower socioeconomic status (SES) households $(n=6 ; 54.6 \%)$ or middle SES households $(n=4 ; 36.4 \%)$. One parent was in a higher SES household (9.0\%). Approximately half $(n=6 ; 54.5 \%)$ of the participants had been previously reported for abuse and neglect to child welfare and nearly half had been previously diagnosed with a mental health and/or substance use disorder $(n=5$; $45.5 \%)$.

\section{Acceptability to Parents}

Ratings of session content indicated that all of the participants gained something positive out of each session, with the majority of participants strongly favoring the content on mindful parenting. In sessions where participants rated that the content raised emotional issues or elicited unwanted thoughts, they also indicated that they gained insight into their experiences during these sessions. Table 2 displays the frequencies of parent ratings of session content. 
Table 2. Participant Ratings of Reactions to Session Content $(n=11)$

\begin{tabular}{|c|c|c|c|c|c|}
\hline Reaction to Session Item & $\begin{array}{c}\text { Strongly } \\
\text { Disagree } \\
\mathrm{n}(\%)\end{array}$ & $\begin{array}{c}\text { Disagree } \\
\mathrm{n}(\%)\end{array}$ & $\begin{array}{c}\text { Neutral } \\
\text { n (\%) }\end{array}$ & $\begin{array}{l}\text { Agree } \\
\mathrm{n}(\%)\end{array}$ & $\begin{array}{c}\text { Strongly } \\
\text { Agree } \\
\mathrm{n}(\%)\end{array}$ \\
\hline \multicolumn{6}{|c|}{ Session 1: Introduction to Mindfulness, Automatic Habits, and Maladaptive Behaviors } \\
\hline Gained something positive & & & & $4(36.4)$ & $7(63.6)$ \\
\hline Raised emotional issues & $4(36.4)$ & $2(18.2)$ & $3(27.3)$ & $2(18.2)$ & \\
\hline Gained insight & & & & $7(63.6)$ & $4(36.4)$ \\
\hline Thought about unwanted things & $3(27.3)$ & $4(36.4)$ & $3(27.3)$ & $1(9.1)$ & \\
\hline \multicolumn{6}{|l|}{ Session 2: Mindful Reappraisal } \\
\hline Gained something positive & & & & $5(45.5)$ & $6(54.5)$ \\
\hline Raised emotional issues & $5(45.5)$ & $2(18.2)$ & $3(27.3)$ & $1(9.1)$ & \\
\hline Gained insight & & & $1(9.1)$ & $6(54.5)$ & $4(36.4)$ \\
\hline Thought about unwanted things & $5(45.5)$ & $3(27.3)$ & $2(18.2)$ & & $1(9.1)$ \\
\hline \multicolumn{6}{|c|}{ Session 3: Savoring Positive Experiences and Interactions with Children } \\
\hline Gained something positive & & & & $5(45.5)$ & $6(54.5)$ \\
\hline Raised emotional issues & $4(36.4)$ & $3(27.3)$ & $2(18.2)$ & $1(9.1)$ & $1(9.1)$ \\
\hline Gained insight & & & $4(36.4)$ & $5(45.5)$ & $2(18.2)$ \\
\hline Thought about unwanted things & $4(36.4)$ & $5(45.5)$ & $2(18.2)$ & & \\
\hline \multicolumn{6}{|c|}{ Session 4: Understanding Impulses and Relationship to Stress } \\
\hline Gained something positive & & & & $5(50.0)$ & $5(50.0)$ \\
\hline Raised emotional issues & $3(30.0)$ & $2(20.0)$ & $2(20.0)$ & $2(20.0)$ & $1(10.0)$ \\
\hline Gained insight & & & $1(10.0)$ & $6(60.0)$ & $3(30.0)$ \\
\hline Thought about unwanted things & $5(50.0)$ & & $2(20.0)$ & $2(20.0)$ & $1(10.0)$ \\
\hline \multicolumn{6}{|l|}{ Session 5: Mindful Parenting } \\
\hline Gained something positive & & & & $3(27.3)$ & $8(72.7)$ \\
\hline Raised emotional issues & $4(36.4)$ & $2(18.2)$ & $2(18.2)$ & $2(18.2)$ & $1(9.1)$ \\
\hline Gained insight & & & & $6(54.5)$ & $5(45.5)$ \\
\hline Thought about unwanted things & $8(72.7)$ & $2(18.2)$ & & $1(9.1)$ & \\
\hline \multicolumn{6}{|c|}{ Session 6: Mindful Planning in the Context of Parenting } \\
\hline Gained something positive & & & & $3(30.0)$ & $7(70.0)$ \\
\hline Raised emotional issues & $2(20.0)$ & $2(20.0)$ & $4(40.0)$ & $2(20.0)$ & \\
\hline Gained insight & & & $1(10.0)$ & $5(50.0)$ & $4(40.0)$ \\
\hline Thought about unwanted things & $6(60.0)$ & $2(20.0)$ & $1(10.0)$ & $1(10.0)$ & \\
\hline
\end{tabular}

Findings from the overall post-program satisfaction questionnaire indicated that the majority of parents felt they benefited from the intervention. Ninety-one percent $(n=10)$ of participants indicated that, "all or most of the time," the program was a big help to them, they got the kind of help through the program they needed, and they learned a lot about how to manage their stress. Moreover, all $(n=11)$ participants reported they enjoyed learning about the concept of mindfulness.

Several themes were identified from the open-ended questions on the program satisfaction questionnaire. Parents reported that the program helped them to (a) recognize triggers to stressful situations, (b) become calmer and more attentive, and (c) improve communication with their child. For example, when asked about what was helpful through participating in the program, one parent stated, “...I learned how to step back and look at a situation, take a deep breath, and not stress about the future... [I could] focus on the here 
and now." Another parent said, "...it helped me to be more aware... and brought to my attention behaviors that I was doing that I didn't like." When asked about how the session content had been applied to interactions with their children, one parent stated, "We are communicating better...not as many screaming matches and not at the level that it used to be." One parent also reported, "I have been able to listen and be more attentive to my daughter." In addition, parents expressed that the "in-home aspect [of the program] was good" and they appreciated the individualized nature of the program. For areas of improvement, parents stated that some of the visual content used to discuss the relationship between substance use and stress could be updated.

\section{Weekly Clinical Trends}

As illustrated in Figure 1, mean score ratings for participants on measures of stress, coping, and mindfulness suggest that the mindfulness-informed intervention was helpful in reducing stress and promoting mindful curiosity and decentering, but questionable for fostering positive coping. Despite subtle decreases in weekly reports of stress across participants, the PND statistic for stress (1.00) indicated that all treatment data points fell below the baseline stress score. Trends illustrate that a greater decrease in stress was reported among participants at the fifth session, but slightly increased by the final session.

Evaluation of the PND statistics for mindful curiosity (1.00) and mindful decentering (1.00) indicated that all data points in the treatment phase exceeded the baseline subscale scores. Trends illustrate that, as a whole, participants reported an increase in awareness and ability to step back from situations without getting taken over by them from baseline. These trends remained predominantly stable until the fifth and sixth sessions at which point there were greater increases in mindfulness. Finally, the PND statistic for the coping subscale (.67) demonstrated that four data points in the treatment phase exceeded the baseline score with the greatest increase at the final session, suggesting that mindfulness-informed training may have been questionably helpful in promoting positive reframing as a coping mechanism for participants.

\section{Discussion}

Findings from the present study suggest that a brief mindfulness-informed intervention for this sample of child-welfare involved parents can be feasibly integrated in public child welfare and is acceptable among parents. In addition, findings suggest that the intervention shows positive changes over time on weekly outcomes, specifically improving state mindfulness and reducing stress, but it may be less useful in increasing the coping skill positive reframing. 

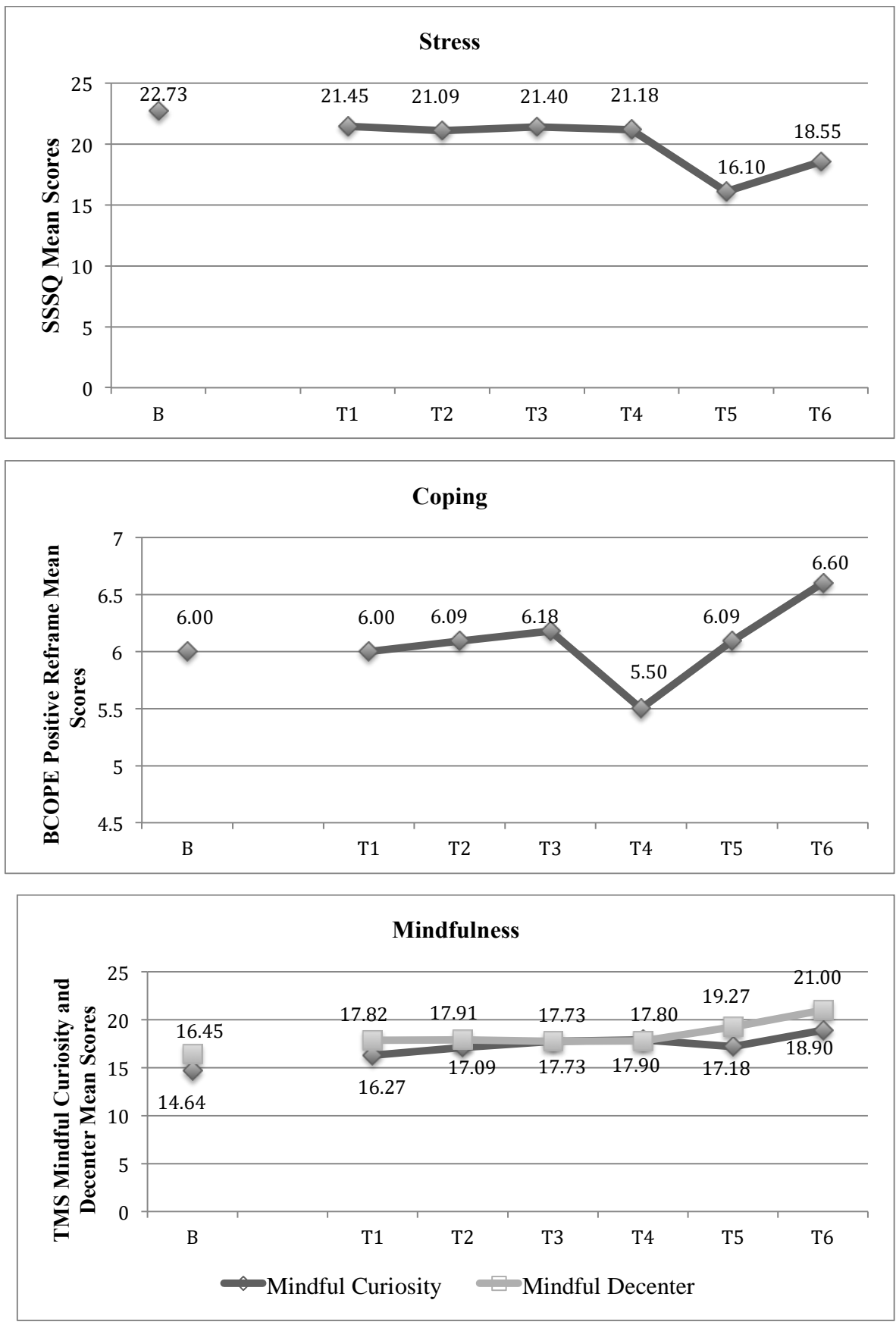

Figure 1. Participant mean scores derived from the Short Stress State Questionnaire Distress Subscale, Brief COPE Positive Reframing Subscale, and Toronto Mindfulness Scale Mindful Curiosity and Mindful Decenter Subscales. 
That the intervention was found to be feasible, as evidenced by recruitment, retention, and treatment attendance rates, may offer a promising approach to address the needs of child welfare-involved families. Of the parents recruited, few dropped out of the intervention, and when they did it was generally due to time constraints or other concerns not related to the intervention itself. With regard to treatment attendance, of the 15 families randomly allocated to the intervention, 11 participated in at least five of the six sessions. Given that this is a challenging population with many barriers to treatment, attrition is in line with prior research using similar samples (e.g., Beasley et al., 2014; Gopalan et al., 2010). Rates of attrition may have been supported by the intentionally brief, flexible, and individualized aspects of the program, as evidence suggests that parents benefit more from programs that are delivered in-home and tailored to meet their unique needs, compared to rigid, group-delivered manual-based programs (Kendall \& Chu, 2000).

The use of a mindfulness-informed intervention for this sample of child welfareinvolved parents with substance misuse was also found to be acceptable. Positive session ratings and qualitative feedback indicated that the intervention was well-received, as parents endorsed multiple benefits of the program. Consistent with previous reports (e.g., Lundahl, Risser, \& Lovejoy, 2006), a number of participants indicated the individualized nature of the program was especially favorable for them such that it allowed for more continuity between sessions compared to their prior experiences in group settings. Moreover, for many participants, the session specifically pertaining to mindful parenting was the most highly rated. Although mindful parenting techniques were infused within each session, parents noted that, from this later session, they gained the most insight into their experiences and received resourceful information from which they could use mindfulness-informed skills as a means to cope with stress in the context of parenting. This suggests that future adaptations to the program should include enhanced content on mindful parenting that is introduced at the start of the program and is a more central focus in additional sessions. This content could potentially replace some of the less preferred content rated by participants. Some of the less preferred content, from the parents' perspective, included the sessions on savoring and understanding the relationship between impulses and stress. Because participants reported many daily stressors, it may have been difficult to broaden their positive emotions in a single session on savoring. Parents also reported increased distress at the third session on savoring and therefore may have been hyper-focused on negative thoughts and feelings. Moreover, in the session that highlighted the relationship between impulses (e.g., use of substances) and stress, participants reported that, in addition to stress, many impulses arose from boredom or social pressure. Adapting this content to include discussions about these differential associations may improve acceptability and would benefit from testing in future studies.

It is important to note that, in light of these findings, the majority of parents involved in the current study identified racially and ethnically as White and therefore may not be representative of the larger child welfare population. Racial and ethnic minority families are often overrepresented in the child welfare system, and research finds that there may be cultural differences regarding their attitudes toward mindfulness practice (Dutton et al., 2013). Notably, researchers have designed mindfulness-based interventions that are culturally suitable for vulnerable populations. For example, Dutton and colleagues (2013) 
conducted focus groups to inform the implementation and enhanced tailoring of Mindfulness-Based Stress Reduction for low-income African-American women with histories of trauma. They found that shorter sessions, diverse applications of both informal and formal mindfulness practice, and clarification of participants' expectations regarding mindfulness practice (e.g., religious beliefs associated with mindfulness) may improve the feasibility and acceptability of mindfulness programs among this population (Dutton et al., 2013). Although some qualities from the mindfulness intervention for child welfareinvolved parents described in this study are consistent with qualities from Dutton and colleagues' (2013) work, their findings have important implications for future research on the feasibility and acceptability as well as adaptation to the current intervention, particularly with a larger and more diverse sample of parents involved in the child welfare system.

Despite questionable results of the intervention on parents' coping skill of positive reframing, it appeared that most parents successfully changed in their weekly stress levels, mindful curiosity (i.e., ability to attend to present moment experiences with a quality of curiosity), and mindful decentering (i.e., awareness of one's experiences without being carried away by them). The initial steps in mindfulness are to increase awareness of where attention gets drawn to and bring it back to the present moment, as well as to note these experiences rather than label or place judgment on them (Bishop et al., 2004). This was a central focus of the mindfulness-informed intervention - particularly attending to thoughts, feelings, and sensations, and recognizing automatic patterns of behavior such as maladaptive parenting and substance misuse - and therefore may support participants' improvements in these specific domains. However, the majority of participants had a substantial increase in their mindfulness curiosity and decentering scores from baseline in sessions five and six, which may indicate that these mindfulness skills required more practice or that the specific content and exercises taught to parents (e.g., mindful parenting, planning for the future) may have been a factor in enhancing parents' ability to better let go of unwanted thoughts, feelings, and sensations. These findings are promising as prior research suggests that brief mindfulness-based training can improve state mindfulness with the potential to spill over into other domains of individual functioning. Specifically, increases in mindfulness have been associated with reduced fatigue, anxiety, and trauma (Kimbrough et al., 2010; Zeidan, Johnson, Diamond, David, \& Goolkasian, 2011), improved affect (Brown \& Ryan, 2003), and better communication (Barnes, Brown, Krusemark, Campbell, \& Rogge, 2007), qualities that may be particularly important in healthy family relationships.

The majority of participants reported slight reductions in stress across treatment sessions, with the greatest decline in stress in session five. Only minor reductions in stress across the other treatment sessions may be attributed to high levels of stress often found in families involved in the child welfare system (Curenton, McWey, \& Bolen, 2009), and the complex, cumulative stressors reported by the participants in this study. For example, many of the participants reported stressors centered on finances, multiple demands of service providers, and interpersonal problems. As these stressors may be more persistent in the lives of child welfare-involved parents and may be exacerbated in particular situations (e.g., receiving a bill in the mail when financial strain already exists), experiencing such 
stress may have contributed to the varying reports of stress across participants. Although there were only slight decreases in stress during the intervention, it is promising that parents also reported improvements in mindfulness such that these skills may have served as potential buffers against new and persistent stressful experiences. Future studies might test the extension of sessions, add additional stress-reduction approaches, or identify other opportunities to practice mindfulness strategies in order to increase the effectiveness of the approach in reducing stress.

Findings should be considered in light of the study's limitations. First, this pilot study included a small homogenous sample, which may in turn limit generalizability of the feasibility, acceptability, and weekly trends of the mindfulness-informed intervention among larger, diverse samples. Second, although the PND metric is a common and systematic approach to evaluate outcomes of single-participant research, the small sample size also reduced statistical power, limiting the opportunity to conduct more traditional and rigorous statistical analyses of the weekly outcome data. Third, as this intervention was designed to reduce participant burden, delivery of content within six sessions may have not allowed for ample time to identify important trends of the program on weekly outcomes of stress, coping, and mindfulness, and may have not provided sufficient time for parents to realize the maximum benefit from the intervention. Fourth, since it was postulated that affecting stress and coping in child welfare-involved parents would lead to positive family functioning outcomes in the long-term, a heterogeneous measure of coping consisting of more than two-item indicators as well as observational data collection methods may have provided more accurate measures on these outcomes. Finally, some dimensions of feasibility and acceptability were not measured in this pilot study, and future research would benefit from including standardized measures of these constructs.

Despite these limitations, this study has several important implications for practice. Stressful life events, including involvement with child welfare, may lead to significant consequences for well-being and parenting. An individual's history of adverse experiences may contribute to automatized and habitual ways of thinking and behaving (Dumas, 2005). For example, the use of behavioral disengagement techniques to cope with stress, such as substance use, may serve to relieve unwanted distress and be implemented without conscious intent. These, often maladaptive, coping strategies may be particularly effective and reinforced because they reduce distress in the immediate term (Sinha, 2001). However, teaching mindfulness to parents has the potential to disrupt automatic, maladaptive coping patterns (e.g., substance use; Bowen et al., 2009) and increase individuals' attention to triggers associated with stressful situations (Creswell \& Lindsay, 2014). When parents have more attentional control over their thoughts, feelings, and sensations, they may be better equipped to respond in a more controlled, rather than reactive, manner even under conditions of stress.

Participating in a brief mindfulness-informed program may in turn facilitate parents' ability to engage in other child welfare-mandated services. Anecdotal reports by participants indicate that mindfulness helped to provide a sense of calmness physiologically as well as increase attention in the present moment. Practicing mindfulness may therefore create a greater capacity to deal with unpleasant situations, and also help parents put aside negative feelings and thoughts associated with the demands of the child 
welfare system. This is particularly well-suited to parents with a history of trauma, which can include physiological problems and difficulty with focus or attending. Mindfulness also has the potential to transfer to parent-child interactions and help parents mindfully attend to their children's behaviors (e.g., Singh et al., 2010), perhaps providing an opportunity to enhance overall family functioning. Teaching mindfulness practice to both parents and children may be another particularly fruitful enhancement to the current intervention, and future studies would benefit from rigorous measurement of parent, child, and family outcomes.

Other adaptations to this mindfulness-informed intervention might include additional mindfulness-informed strategies that also promote the use of alternative emotional, cognitive, and behavioral coping strategies. Given the types of stressors families faced (e.g., finances, pressure from service providers, interpersonal problems), practical guidelines for handling these stressors and refinement of the intervention to meet the individual needs of families may also be a favorable enhancement.

Taken together, the data indicate initial support for the use of a brief mindfulnessinformed intervention for child welfare-involved families with substance misuse. This study adds to the growing body of literature on mindfulness practice such that it may be a useful and feasible approach to integrate within public child welfare settings. However, given the range of stressors likely experienced by participants in the current study and the variability in affecting the domain of coping, mindfulness-informed training may better help families reach their full potential if it is delivered in conjunction with other therapeutic skill-building programs. An integrated mindfulness intervention for targeting stress and improving coping may in turn better serve as a trauma-informed approach and impact longterm family functioning for child welfare-involved families with substance misuse.

\section{References}

Barnes, S., Brown, K. W., Krusemark, E., Campbell, W. K., \& Rogge, R. D. (2007). The role of mindfulness in romantic relationship satisfaction and responses to relationship stress. Journal of Marital and Family Therapy, 33(4), 482-500. doi:https://doi.org/10.1111/j.1752-0606.2007.00033.x

Beasley, L. O., Silovsky, J. F., Owora, A., Burris, L., Hecht, D., DeMoraes-Huffine, P.,...Tolma, E. (2014). Mixed-methods feasibility study on the cultural adaptation of a child abuse prevention model. Child Abuse \& Neglect, 38(9), 1496-1507. doi:https://doi.org/10.1016/j.chiabu.2014.04.017

Benotsch, E. G., Brailey, K., Vasterling, J. J., Uddo, M., Constans, J. I., \& Sutker, P. B. (2000). War zone stress, personal and environmental resources, and PTSD symptoms in Gulf War veterans: A longitudinal perspective. Journal of Abnormal Psychology, 109(2), 205-213. doi:https://doi.org/10.1037/0021-843X.109.2.205

Bishop, S. R., Lau, M., Shapiro, S., Carlson, L., Anderson, N. D., Carmody, J., ... Devins, G. (2004). Mindfulness: A proposed operational definition. Clinical Psychology: Science and Practice, 11, 230-241. doi:https://doi.org/10.1093/clipsy.bph077 
Bowen, S., Chawla, N., Collins, S. E., Witkiewitz, K., Hsu, S., Grow, J., \& Marlatt, A. (2009). Mindfulness-based relapse prevention for substance use disorders: A pilot efficacy trial. Substance Abuse, 30, 295-305. doi:https://doi.org/10.1080/08897070903250084

Brown, K. W., \& Ryan, R. M. (2003). The benefits of being present: Mindfulness and its role in psychological well-being. Journal of Personality and Social Psychology, 84, 822-848. doi:https://doi.org/10.1037/0022-3514.84.4.822

Carlson, L. E., Speca, M., Patel, K. D., \& Goodey, E. (2004). Mindfulness-based stress reduction in relation to quality of life, mood, symptoms of stress and levels of cortisol, dehydroepiandrosterone sulfate (DHEAS) and melatonin in breast and prostate cancer outpatients. Psychoneuroendocrinology, 29(4), 448-474. doi:https://doi.org/10.1016/S0306-4530(03)00054-4

Carver, C. S. (1997). You want to measure coping but your protocol' too long: Consider the Brief COPE. International Journal of Behavioral Medicine, 4(1), 92-100. doi:https://doi.org/10.1207/s15327558ijbm0401_6

Cloitre, M., Stolbach, B. C., Herman, J. L., van der Kolk, B., Pynoos, R., \& Wang, J. (2009). A developmental approach to complex PTSD: Childhood and adult cumulative trauma as predictors of symptom complexity. Journal of Traumatic Stress,22(5), 399-408. doi:https://doi.org/10.1002/jts.20444

Crabtree, B. F., \& Miller, W. L. (1999). Doing qualitative research. Thousand Oaks, CA: Sage.

Creswell, J. D., \& Lindsay, E. K. (2014). How does mindfulness training affect health? A mindfulness stress buffering account. Current Directions in Psychological Science, 23(6), 401-407. doi:doi:https://doi.org/10.1177/0963721414547415

Curenton, S., McWey, L., \& Bolen, M. (2009). Distinguishing maltreating versus nonmaltreating at-risk families: A discriminant function analysis with implications for foster care and early childhood education interventions. Families in Society: The Journal of Contemporary Social Services, 90(2), 176-182. doi:https://doi.org/10.1606/1044-3894.3871

Davis, K. M., Lau, M. A., \& Cairns, D. R. (2009). Development and preliminary validation of a trait version of the Toronto Mindfulness Scale. Journal of Cognitive Psychotherapy, 23(3), 185-197. doi:https://doi.org/10.1891/0889-8391.23.3.185

Dumas, J. E. (2005). Mindfulness-based parent training: Strategies to lessen the grip of automaticity in families with disruptive children. Journal of Clinical Child \& Adolescent Psychology, 34(4), 779-791. doi:https://doi.org/10.1207/s15374424jccp3404_20

Duncan, L. G., Coatsworth, J. D., \& Greenberg, M. T. (2009a). A model of mindful parenting: Implications for parent-child relationships and prevention research. Clinical Child and Family Psychology Review, 12(3), 255-270. doi:https://doi.org/10.1007/s10567-009-0046-3 
Duncan, L. G., Coatsworth, J. D., \& Greenberg, M. T. (2009b). Pilot study to gauge acceptability of a mindfulness-based, family-focused preventive intervention. The Journal of Primary Prevention, 30(5), 605-618. doi:https://doi.org/10.1007/s10935009-0185-9

Dutton, M. A., Bermudez, D., Matas, A., Mjid, H., \& Myers, N. (2013). Mindfulnessbased stress reduction for low-income, predominantly African American women with PTSD and a history of intimate partner violence. Cognitive Behavioral Practice, 20, 23-32. doi:https://doi.org/10.1016/j.cbpra.2011.08.003

Follette, V., Palm, K. M., \& Pearson, A. N. (2006). Mindfulness and trauma: Implications for treatment. Journal of Rational-Emotive and Cognitive-Behavior Therapy, 24, 4561. doi:https://doi.org/10.1007/s10942-006-0025-2

Garland, E. L. (2013). Mindfulness-oriented recovery enhancement for addiction, stress, and pain. Washington, D.C.: NASW Press.

Garland, E. L., Gaylord, S. A., Boettiger, C. A., \& Howard, M. O. (2010). Mindfulness training modifies cognitive, affective, and physiological mechanisms implicated in alcohol dependence: Results from a randomized controlled pilot trial. Journal of Psychoactive Drugs, 42, 177-192. doi:https://doi.org/10.1080/02791072.2010.10400690

Gopalan, G., Goldstein, L., Klingenstein, K., Sicher, C., Blake, C., \& McKay, M. M. (2010). Engaging families into child mental health treatment: Updates and special considerations. Journal of the Canadian Academy of Child and Adolescent Psychiatry, 19(30), 182-196.

Harris-McKoy, D., Meyer, A. S., McWey, L. M., \& Henderson, T. L. (2014). Substance use, policy, and foster care. Journal of Family Issues, 35(10), 1298-1321. doi:https://doi.org/10.1177/0192513X13481439

Helton, W. S. (2004). Validation of a Short Stress State Questionnaire. Proceedings of the Human Factors and Ergonomics Society Annual Meeting, 48(11), 1238-1242. doi:https://doi.org/10.1177/154193120404801107

Hillson, J. M. C., \& Kuiper, N. A. (1994). A stress and coping model of child maltreatment. Clinical Psychology Review, 14(4), 261-285. doi:https://doi.org/10.1016/0272-7358(94)90025-6

Kabat-Zinn, J. (1990). Full catastrophe living. NY: Delacorte Press.

Kabat-Zinn, J. (1994). Wherever you go, there you are: Mindfulness meditation in everyday life. New York, NY: Hyperion.

Kabat-Zinn, J. (2015). Mindfulness, healing, and transformation. Eau Claire, WI: Psychotherapy Networker \& PESI, Inc.

Kabat-Zinn, M., \& Kabat-Zinn, J. (1997). Everyday blessings: The inner work of mindful parenting. New York, NY: Hyperion. 
Kendall, P. C., \& Chu, B. C. (2000). Retrospective self-reports of therapist flexibility in a manual-based treatment for youths with anxiety disorders. Journal of Clinical Child Psychology, 29(2), 209-220. doi:https://doi.org/10.1207/S15374424jccp2902_7

Kimbrough, E. Magyari, T., Langenberg, P., Chesney, M., \& Berman, B. (2010). Mindfulness intervention for child abuse survivors. Journal of Clinical Psychology, 66(1), 17-33.

King, A. P., Erickson, T. M., Giardino, N. D., Favorite, T., Rauch, S. A. M., Robinson, E.,... Lberzon, I. (2013). A pilot study of group mindfulness-based cognitive therapy (MBCT) for combat veterans with posttraumatic stress disorder (PTSD). Depression and Anxiety, 30(7), 638-645. doi:https://doi.org/10.1002/da.22104

Lau, M. A., Bishop, S. R., Segal, Z. V., Buis, T., Anderson, N. D., Carlson, L., ... Devins, G. (2006). The Toronto Mindfulness Scale: Development and validation. Journal of Clinical Psychology, 62, 1445-1467. doi:https://doi.org/10.1002/jclp.20326

Littell, J., Alexander, L., \& Reynolds, W. (2001). Client participation: Central and underinvestigated elements of intervention. Social Service Review, 75, 1-28. doi:https://doi.org/10.1086/591880

Littell, J. H., \& Tajima, E. A. (2000). A multilevel model of client participation in intensive family preservation services. Social Service Review, 74, 405-435. doi:https://doi.org/10.1086/516411

Lundahl, B., Risser, H., \& Lovejoy, M. (2006). A meta-analysis of parent training: Moderators and follow-up effects. Clinical Psychology Review, 26(1), 86-104. doi:https://doi.org/10.1016/j.cpr.2005.07.004

National Center on Addiction and Substance Abuse at Columbia University. (2005). Family matters: Substance abuse and the American family. Retrieved from http://www.casacolumbia.org/addiction-research/reports/family-matters-substanceabuse-and-american-family

National Child Traumatic Stress Network. (2011). Birth parents with trauma histories and the child welfare system: A guide for child welfare staff. Retrieved from http://nctsn.org/sites/default/files/assets/pdfs/birth parents trauma history fact shee t_final.pdf

Oliver, J. E. (1993). Intergenerational transmission of child abuse: Rates, research, and clinical implications. American Journal of Psychiatry, 150, 1315-1324. doi:https://doi.org/10.1176/ajp.150.9.1315

Patterson, R. G. (1982). A social learning approach: Coercive family process (Vol. 3). Eugene, OR: Castalia.

Scruggs, T. E., \& Mastropieri, M. A. (1998). Synthesizing single subject research: Issues and applications. Behavior Modification, 22, 221-242. doi:https://doi.org/10.1177/01454455980223001 
Scruggs, T. E., Mastropieri, M. A., \& Casto, G. (1987). The quantitative synthesis of single subject research: Methodology and validation. Remedial and Special Education, 8, 24-33. doi:https://doi.org/10.1177/074193258700800206

Segal, Z., V., Williams, J. M. G., \& Teasdale, J. D. (2002). Mindfulness-based cognitive therapy for depression: A new approach to preventing relapse. New York: Guilford.

Singh, N. N., Lancioni, G. E., Winton, A. S. W., Singh, J., Singh, A. N., Adkins, A. D., \& Wahler, R. G. (2010). Training in mindful caregiving transfers to parent-child interactions. Journal of Child and Family Studies, 19(2), 167-174. doi:https://doi.org/10.1007/s10826-009-9267-9

Sinha, R. (2001). How does stress increase risk of drug abuse and relapse? Psychopharmacology, 158(4), 343-359. doi:https://doi.org/10.1007/s002130100917

U.S. Department of Health and Human Services, National Clearinghouse on Child Abuse and Neglect. (2003). Substance abuse and child maltreatment. Retrieved from http://nccanch.acf.hhs.go

Weinstein, N., Brown, K. W., \& Ryan, R. M. (2009). A multi-method examination of the effects of mindfulness on stress attribution, coping, and emotional well-being. Journal of Research in Personality, 43(3), 374-385. doi:https://doi.org/10.1016/j.jrp.2008.12.008

Williams, K. A., Kolar, M. M., Reger, B. E., \& Pearson, J. C. (2001). Evaluation of a wellness-based mindfulness stress reduction intervention: A controlled trial. American Journal of Health Promotion, 15(6), 422-432. doi:https://doi.org/10.4278/0890-1171-15.6.422

Yusoff, N., Low, W. Y., \& Yip, C. H. (2010). Reliability and validity of the Brief COPE scale (English version) among women with breast cancer undergoing treatment of adjuvant chemotherapy: A Malaysian study. Malaysian Journal of Psychiatry, 65, 41-44.

Zeidan, F., Johnson, S. K., Diamond, B. J., David, Z., \& Goolkasian, P. (2011). Mindfulness meditation improves cognition: Evidence of brief mental training. Conscious Cognition, 19, 597-605. doi:https://doi.org/10.1016/j.concog.2010.03.014

Author note: Address correspondence to: Samantha M. Brown, Department of Psychology, University of Denver, 2155 S. Race Street, Denver, CO 80211, 303-8717774, Samantha.M.Brown@ucdenver.edu

Acknowledgements: Funding for this study was provided by the New York Community Trust Fahs-Beck Fund for Research and Experimentation, the American Psychological Association Section on Child Maltreatment, and the Society for Social Work and Research. 